\title{
On the Relationship Between SAS Image Resolution and Target-Detection Performance
}

\author{
David P. Williams \\ NATO STO CMRE \\ La Spezia, Italy \\ david.williams@cmre.nato.int
}

\author{
Alan J. Hunter \\ University of Bath \\ Bath, United Kingdom \\ a.j.hunter@bath.ac.uk
}

\begin{abstract}
The relationship between synthetic aperture sonar (SAS) image resolution and target-detection performance is quantified. It is first demonstrated how a lower-resolution SAS system can be simulated in a principled manner by bandlimiting the image wavenumber spectrum of a full-resolution SAS image. This insight enables a direct comparison of targetdetection performance in imagery of different resolutions while all other variables (such as data-collection conditions) remain fixed. The comparison is achieved by degrading a given SAS image to a series of different along-track resolutions and range resolutions, and then applying the same detection algorithm to the resulting set of images. This is repeated on a large scale for a set of real SAS data collected at sea at eight different geographical locations in various environmental conditions; the data considered comprise over 1500 images collectively spanning over 8 square-kilometers of seabed and containing over 2000 mine-like targets. The results quantify the dependence of target-detection performance and false alarm rate on alongtrack resolution and range resolution. This information can be used to determine the minimum processing requirements needed onboard an autonomous underwater vehicle for effective through-the-sensor survey adaptation, and also to inform SAS system design and procurement decisions.
\end{abstract}

\section{INTRODUCTION}

The high-resolution imaging of underwater environments afforded by sonar has proven particularly useful in diverse applications, from archaeology [1] and habitat mapping [2] to pipeline inspection [3] and mine detection [4]. But increasingly, the traditional side-scan sonar is being supplanted by synthetic aperture sonar (SAS) systems, which provide even higher resolution imagery, though also at a higher cost. This resolution/cost tradeoff raises an obvious question: For the application under investigation, what is the expected gain in performance when using SAS instead of a simple side-scan sonar? Providing a quantitative answer to this query is not a trivial task, but it is necessary for making informed sonarsystem procurement decisions.

Meanwhile, the maturation of underwater robot technology means that these sophisticated SAS systems are increasingly deployed on an autonomous underwater vehicle (AUV). Because of the cost and time-sensitive nature of at-sea surveys, the next priority is to embed intelligence in the AUV so that it can immediately react to the data it collects [5]. By adapting its survey route in situ and efficiently allocating resources, the AUV can collect the most informative data for the task at hand while simultaneously reducing mission times. However, constraints on the available processing power onboard an AUV mean that reduced-resolution imagery is often a necessary concession in order to maintain real-time processing. For this reason, it is also important to know the level of performance degradation that would be expected if data were processed at a lower resolution.

Motivated by these questions, we seek to experimentally quantify the relationship between SAS image resolution and performance. Specifically, we address this problem in the context of a mine countermeasures application in which performance is measured in terms of target-detection capability (and false alarm rate). That is, how does target-detection performance vary when the SAS data is processed at different levels of resolution?

The remainder of this paper is organized as follows. In Sec. II, we demonstrate how lower-resolution SAS imagery can be generated in a principled manner from full-resolution imagery. Sec. III describes the real, measured SAS data collected at sea that are used for the experiments, the results of which are shown in Sec. IV. A brief discussion about sonar system comparisons appears in Sec. V, before concluding remarks and directions for future work are given in Sec. VI.

\section{SAS IMAGE RESOLUTION}

Synthetic aperture sonar (SAS) works by coherently summing received acoustic signals of overlapping elements in an array, and it provides an order-of-magnitude improvement in resolution over simple (real aperture) side-scan sonar data [6]. The resulting high-resolution SAS imagery provides a detailed view of the seafloor that makes detection of proud targets (e.g., mines) possible. One example SAS image is shown in Fig. 1.

To investigate the effects of image resolution on targetdetection performance, we use data from a high-resolution SAS and degrade its resolution in varying degrees via the following procedure. (It should be noted that a related method for multi-aspect processing was described in [7].)

The full image resolution in the along-track and range dimensions are related, respectively, to the spatial and temporal bandwidths of the sonar by $\delta_{x}=D / 2$ and $\delta_{y}=c / 2 B$, where $D$ is the along-track dimension of the largest (transducer) aperture, $B$ is the signal bandwidth in $\mathrm{Hz}$, and $c \approx 1500 \mathrm{~m} / \mathrm{s}$ is the propagation speed. 


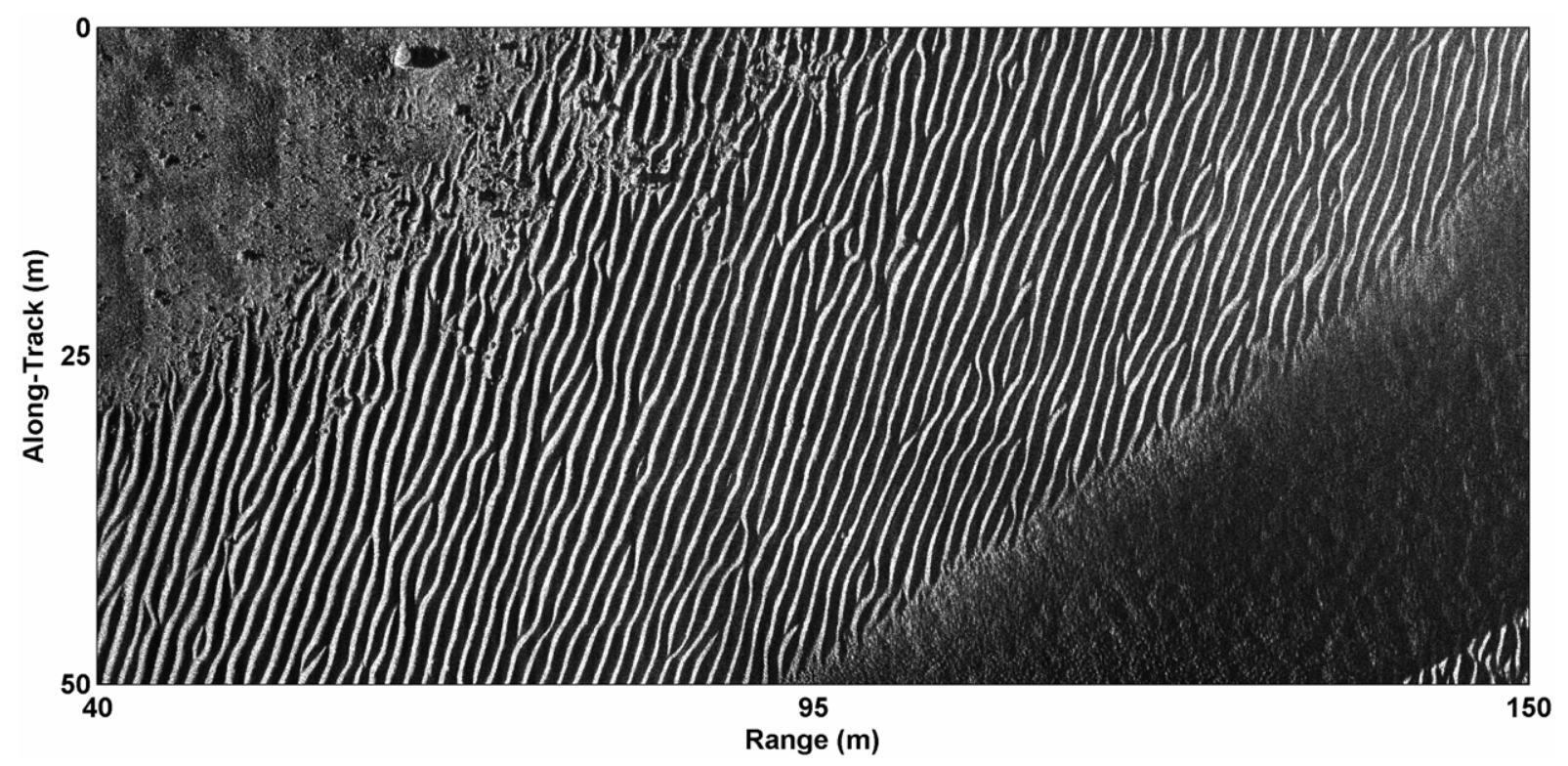

Fig. 1. An example SAS image.

We degrade the resolution by factors of $\alpha_{x}$ and $\alpha_{y}$ in each of the respective image dimensions by band-limiting the image wavenumber spectrum, i.e.,

$$
i^{\prime}(x, y)=\int_{-\infty}^{\infty} I^{\prime}\left(k_{x}, k_{y}\right) \exp \left(j\left(k_{x} x+k_{y} y\right)\right) \mathrm{d} k_{x} \mathrm{~d} k_{y},
$$

where

$$
I^{\prime}\left(k_{x}, k_{y}\right)=\operatorname{win}\left(\frac{\delta_{x}^{\prime}}{2 \pi} k_{x}\right) \operatorname{win}\left(\frac{\delta_{y}^{\prime}}{2 \pi} k_{y}\right) I\left(k_{x}, k_{y}\right)
$$

is the band-limited spectrum with wavenumbers $k_{x}$ and $k_{y}$, $\delta_{x}^{\prime}=\alpha_{x} \delta_{x}$ and $\delta_{y}^{\prime}=\alpha_{y} \delta_{y}$ are the dimensions of the degraded resolution cell, win $(x)$ is a window function that is non-zero only on the interval $x \in[-0.5,0.5]$, and

$$
I\left(k_{x}, k_{y}\right)=\int_{-\infty}^{\infty} i(x, y) \exp \left(-j\left(k_{x} x+k_{y} y\right)\right) \mathrm{d} k_{x} \mathrm{~d} k_{y}
$$

is the spectrum of the full resolution image $i(x, y)$. We then decimate the image pixels accordingly by the same factors $\alpha_{x}$ and $\alpha_{y}$.

The effects of this image wavenumber filtering procedure are illustrated for a given SAS image in Fig. 2, where the band-limited spectra for various degraded-resolution cases are shown. (For purposes of illustration, the spectra are of a small snippet that was extracted from a large SAS scene.) Importantly, this principled approach is not equivalent to simply downsampling the full-resolution image, which would not truly simulate a lower-resolution system in the proper manner.

An example result of degrading a full-resolution SAS image (snippet) to a series of different along-track resolutions and range resolutions is shown in Fig. 3. (The image wavenumber spectra shown in Fig. 2 correspond to this target.)

\section{DATA}

In this work, the procedure described in Sec. II is exploited to quantify target-detection performance as a function of image resolution. The data used in the study were collected by the CMRE's SAS-equipped AUV called MUSCLE. This SAS has an aperture length of $D=5 \mathrm{~cm}$ and bandwidth of $B=60 \mathrm{kHz}$, which yields a (full) resolution cell of $\delta_{x}=2.5 \mathrm{~cm}$ in the along-track dimension and $\delta_{y}=1.25 \mathrm{~cm}$ in the range dimension.

The data were collected during major sea experiments conducted by NURC/CMRE between 2008 and 2012 at eight different geographical sites in the Mediterranean and Baltic Seas. The data comprise 1527 images (of which Fig. 1 is one) collectively spanning over $8 \mathrm{~km}^{2}$ of seabed and containing over 2157 mine-like targets.

Here, each full-resolution image in the data set is degraded to a series of different along-track resolutions and range resolutions. We consider the cases when the image-resolution degradation factors $\alpha_{x}$ and $\alpha_{y}$ are each taken from the set $A=\{1,2,4,6,8,10\}$. This translates into examining 36 total cases where the along-track resolutions considered are $2.5 \mathrm{~cm}$ to $25.0 \mathrm{~cm}$, by factors of 2 , and the range resolutions considered are $1.25 \mathrm{~cm}$ to $12.5 \mathrm{~cm}$, by factors of 2 .

A cascaded target-detection algorithm [8] is then applied to every image. The algorithm is based on an integralimage formulation that allows for the very rapid search of shadow regions and highlight regions that together are often indicative of targets. This detection algorithm automatically adjusts necessary size-based quantities according to the (input) image resolution, but all other algorithm parameters were kept fixed for all experiments.

\section{EXPERIMENTAL RESULTS}

For each image-resolution case, the numbers of targets correctly detected and false alarms generated are computed. 


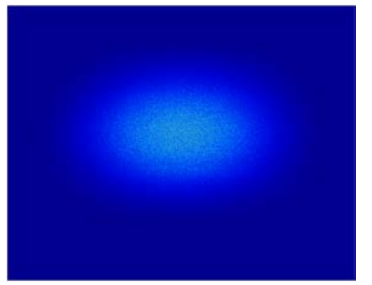

(a)

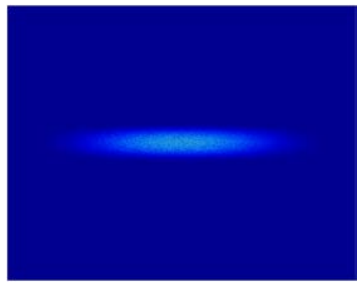

(b)

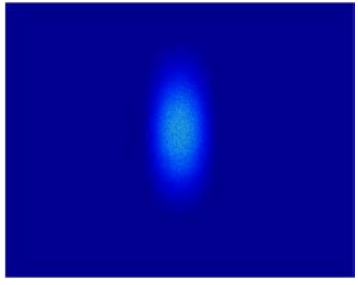

(c)

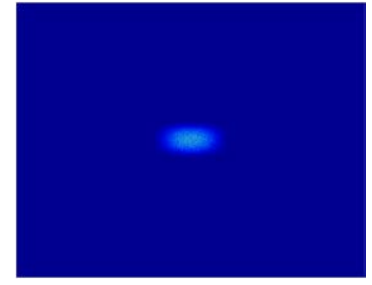

(d)

Fig. 2. For a given SAS image, the image wavenumber spectra where the $x$-axis corresponds to wavenumber $k_{x}$ and the $y$-axis corresponds to wavenumber $k_{y}$ for (a) the original full-resolution image $\left(\alpha_{x}=1\right.$ and $\left.\alpha_{y}=1\right)$; (b) the image band-limited in $k_{y}$, such that the range resolution is degraded by a factor of $\alpha_{y}=6$; (c) the image band-limited in $k_{x}$, such that the along-track resolution is degraded by a factor of $\alpha_{x}=6$; and (d) the image band-limited in both $k_{y}$ and $k_{x}$, such that the range and along-track resolutions are each degraded by a factor of 6 .

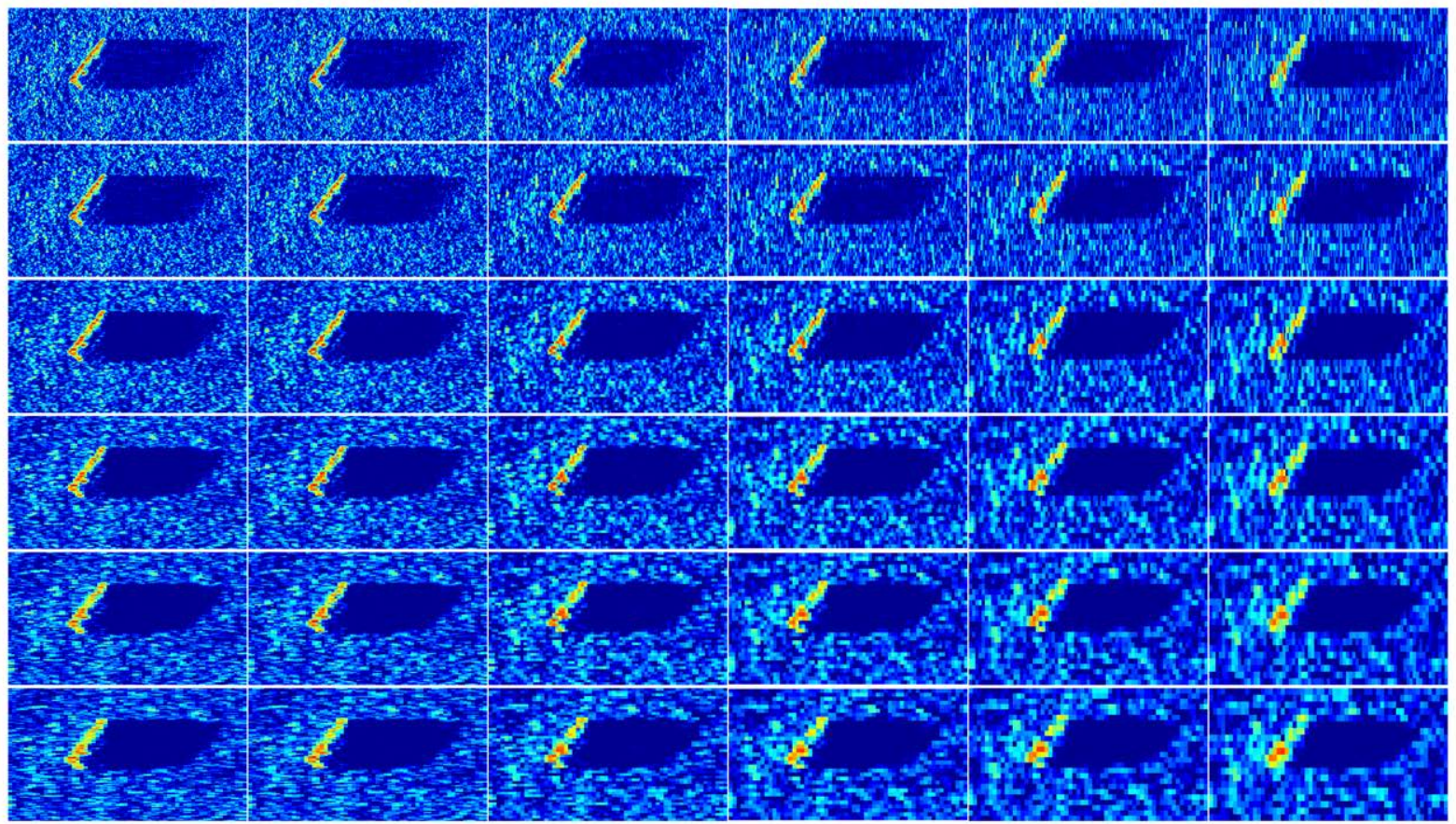

Fig. 3. A target in SAS images at different along-track and range resolutions, where the $y$-axis corresponds to along-track direction and the $x$-axis corresponds to range. Each image covers an area of $5 \mathrm{~m} \times 9 \mathrm{~m}$. The full-resolution image, in the upper left, has an along-track resolution of $2.5 \mathrm{~cm}$ and a range resolution of $1.25 \mathrm{~cm}$. Each column, from left to right, shows the image with the along-track resolution degraded by a factor of $1,2,4,6,8$, and 10 , respectively. Each row, from top to bottom, shows the image with the range resolution degraded by a factor of $1,2,4$, 6,8 , and 10 , respectively.

A summary of these detection performance results across all sites is shown in Fig. 4. It should be noted that the results (e.g., probability of detection) are presented with respect to the full-resolution case. As one would expect, the probability of detection decreases and the false alarm rate increases as resolution degrades. However, importantly we quantify what these changes are for this target-detection task.

For example, when $\alpha_{x}=4$ and $\alpha_{y}=4$, corresponding to an along-track resolution of $10.0 \mathrm{~cm}$ and a range resolution of $5.0 \mathrm{~cm}$, the probability of detection degrades by about $3 \%$ while the number of false alarms grows by a factor of about 1.5. (It is interesting to note that the approximate resolution of one commonly used $900 \mathrm{kHz}$ side-scan sonar is $11.7 \mathrm{~cm}$ in the along-track direction and $5.8 \mathrm{~cm}$ in the range direction.) These results can begin to partially answer some of the questions posed in Sec. I.

The results from Fig. 4 are also presented in an alternative format in Fig. 5, which provides a clearer picture of the general trend in performance as a function of the product of along-track and range resolution factors. This product multiplied by $\delta_{x} \delta_{y}=3.125 \mathrm{~cm}^{2}$ gives the area of the resolution cell, which is also inversely proportional to the number of pixels on target. For Fig. 5, when multiple cases produce the same product (e.g., a product of 6 is produced by both $\left\{\alpha_{x}=6, \alpha_{y}=1\right\}$ and $\left\{\alpha_{x}=1, \alpha_{y}=6\right\}$ ), the result shown is the average.

\section{Discussion}

A topic that frequently arises in the underwater mine countermeasures community is the desire to compare the performance of different sonar systems. In particular, there is often interest in comparing a side-scan sonar on one AUV to a SAS on some other AUV. Unfortunately, it is impossible to do a straightforward, head-to-head comparison that can 


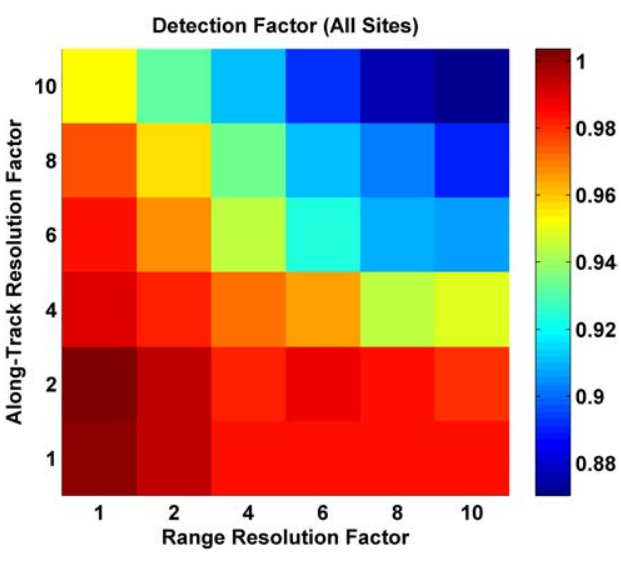

(a)

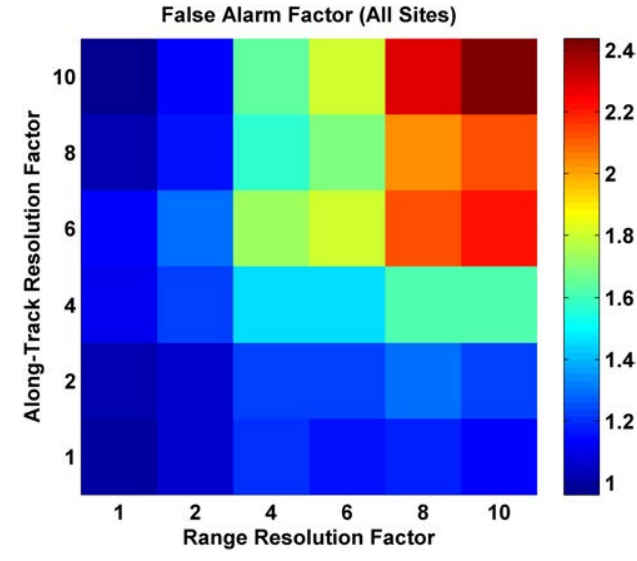

(b)

Fig. 4. Detection performance, across all sites, as a function of along-track and range resolution factors. (a) The detection factor expresses the probability of detection divided by that of the full-resolution case. (b) The false alarm factor expresses the number of false alarms divided by that of the full-resolution case (e.g., a false alarm factor of 2 indicates the case had twice as many false alarms as the full-resolution case).

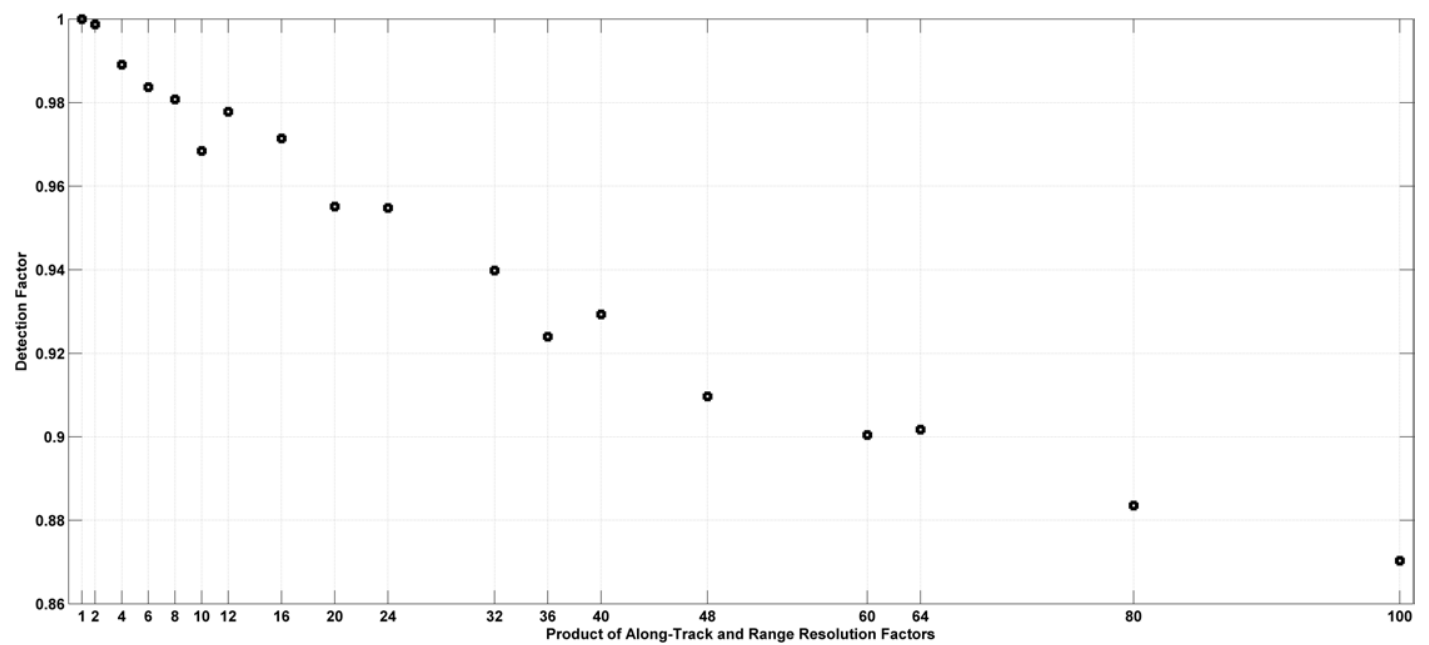

(a)

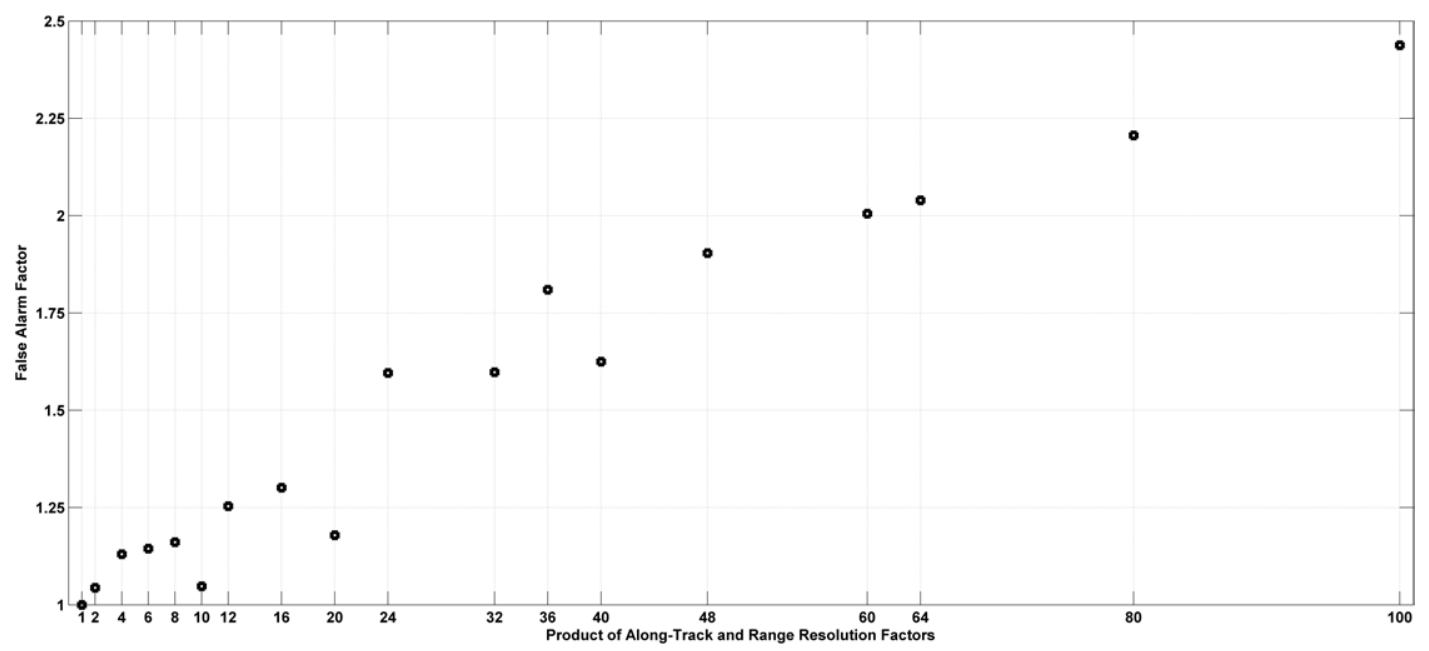

(b)

Fig. 5. Detection performance, across all sites, as a function of the product of along-track and range resolution factors. (a) The detection factor expresses the probability of detection divided by that of the full-resolution case. (b) The false alarm factor expresses the number of false alarms divided by that of the full-resolution case. 
result in a simple numerical answer.

Even if the two systems survey the same area with very little temporal offset, it is not possible to control the numerous factors that impact performance. The underwater environment is constantly changing - currents are flowing, fish are passing through the scene, etc. - but even larger obstacles exist. First, the platforms on which the sonar systems are deployed will have different stabilities and navigation capabilities, which can impact the quality of the throughthe-sonar data collected. But the sonar systems will usually also be flown at different altitudes, which in turn impacts the incidence angles of the signals. Moreover, the systems will also insonify different swaths of seafloor, so a common survey track executed by each AUV will not produce imagery over the same exact area of seafloor. The area coverage rates of each system will also differ, a fact that highlights the need for an additional assessment metric. In addition, the along-track resolution of a side-scan sonar is not constant in range, unlike a SAS. All of these basic issues make it very difficult to isolate the fundamental effects of the sonar itself on performance.

This highlights the utility of the present study, in which all other conditions are kept constant. The only factor that can affect performance here is the image resolution. The aforementioned issues with side-scan sonar data mean that the results based on the lower-resolution data simulated in this work should be views as an upper bound on what can be expected to be achieved with a real side-scan system at the given resolution.

In this work, the impact of image resolution on targetdetection performance was examined. No consideration was given to the subsequent stages in the target-recognition chain: feature extraction and classification. In general, the detection stage relies on relatively coarse attributes, whereas the classification stage typically exploits finer details. As a result, it is likely that the use of lower-resolution imagery will degrade classification performance more severely than it did to detection performance. Nevertheless, this study provides a useful initial investigation into comparing image resolution and performance, and in turn provides quantitative evidence of the relationship. The results obtained here can be extrapolated to existing side-scan sonar systems as an estimate for purposes of performance comparison.

\section{CONCLUSION}

To our knowledge, this work is the first to quantify targetdetection performance of SAS systems as a function of varying resolution. Because increases in temporal bandwidth and decreases in spatial bandwidth (i.e., transducer element size) increase the cost of a SAS system, it is valuable to know the extent to which target-detection performance is affected by image resolution. This information can be exploited to inform SAS system design and procurement decisions. Additionally, it can help determine the minimum resolution to which images should be processed onboard an AUV - where processing capability is limited - for effective real-time survey adaptation based on through-the-sensor data.

Future work will investigate the impact of SAS image resolution on the feature-extraction process and on classification. Other work will explore the development of new features based on multi-resolution imagery.

\section{REFERENCES}

[1] H. Singh, J. Adams, D. Mindell, and B. Foley, "Imaging underwater for archaeology," Journal of Field Archaeology, vol. 27, no. 3, pp. 319-328, 2000.

[2] S. Williams, O. Pizarro, M. Jakuba, and N. Barrett, "AUV benthic habitat mapping in south eastern Tasmania," Field and Service Robotics, vol. 62, pp. 275-284, 2010.

[3] Y. Petillot, S. Reed, and J. Bell, "Real time AUV pipeline detection and tracking using side scan sonar and multi-beam echo-sounder," in Proc. IEEE OCEANS, 2002, pp. 217-222.

[4] S. Reed, I. Ruiz, C. Capus, and Y. Petillot, "The fusion of large scale classified sidescan sonar image mosaics," IEEE Transactions on Image Processing, vol. 15, no. 7, pp. 2049-2060, 2006.

[5] D. Williams, A. Vermeij, F. Baralli, J. Groen, and W. Fox, "In situ AUV survey adaptation using through-the-sensor sonar data," in Proc. International Conference on Acoustics, Speech, and Signal Processing, 2012, pp. 2525-2528.

[6] M. Hayes and P. Gough, "Broad-band synthetic aperture sonar," IEEE Journal of Oceanic Engineering, vol. 17, no. 1, pp. 80-94, 1992.

[7] J. Fernandez and J. Christoff, "Multi-aspect synthetic aperture sonar," in Proc. IEEE OCEANS, 2000, pp. 177-180.

[8] D. Williams, "Fast target detection in synthetic aperture sonar imagery: A new algorithm and large-scale performance analysis," IEEE Journal of Oceanic Engineering, vol. 40, no. 1, pp. 71-92, 2015. 